Annuaire du Collège de France 2017-2018

\title{
Dynamiques du vivant
}

\section{Thomas Lecuit}

\section{OpenEdition}

Journals

Édition électronique

URL : https://journals.openedition.org/annuaire-cdf/15568

DOI : 10.4000/annuaire-cdf.15568

ISBN : 978-2-7226-0572-5

ISSN : 2109-9227

Éditeur

Collège de France

Édition imprimée

Date de publication : 30 décembre 2020

Pagination : 139-143

ISBN : 978-2-7226-0516-9

ISSN : 0069-5580

\section{Référence électronique}

Thomas Lecuit, « Dynamiques du vivant », L'annuaire du Collège de France [En ligne], 118 | 2020, mis en ligne le 01 avril 2021, consulté le 22 août 2022. URL : http://journals.openedition.org/annuaire-cdf/ 15568 ; DOI : https://doi.org/10.4000/annuaire-cdf.15568 


\title{
DYNAMIQUES DU VIVANT
}

\author{
Thomas LECUIT \\ Membre de l'Institut (Académie des sciences), \\ professeur au Collège de France
}

Mots-clés : morphogenèse, adhésion, tension cellulaire

La série de cours "Mécanique de la morphogenèse : principes fondamentaux » est disponible, en audio et vidéo, sur le site internet du Collège de France (https://www. college-de-france.fr/site/thomas-lecuit/course-2017-2018.htm), ainsi que le colloque "Mécanique cellulaire et tissulaire » (https://www.college-de-france.fr/site/thomaslecuit/symposium-2017-2018.htm).

\section{ENSEIGNEMENT}

\section{COURS - MÉCANIQUE DE LA MORPHOGENĖSE : PRINCIPES FONDAMENTAUX}

\section{Introduction générale}

Le cours 2017-2018, « Mécanique de la morphogenèse », initie le thème des bases moléculaires, cellulaires et biophysiques des formes tissulaires chez les animaux et les plantes, qui sera poursuivi l'an prochain. En s'inscrivant dans une démarche historique des concepts clés, il rendra compte des avancées scientifiques décisives permettant de comprendre le paradoxe apparent de structures tissulaires (organes, embryons) à l'organisation robuste, et néanmoins incessamment remodelées.

Le cours a développé, à partir de la littérature scientifique ancienne et très récente, les fondements de la cohésion tissulaire chez les animaux, à rechercher dans les propriétés tout à fait remarquables de l'adhérence biologique, et de sa plasticité, dont le ressort principal est la contractilité cellulaire.

\section{Cours 1 - Introduction : organisation et plasticité tissulaires}

Ce premier cours se veut une introduction générale à la thématique de la Mécanique de la morphogenèse. Illustrant la diversité de l'espace des formes, nous 
exposons l'enjeu d'identifier des principes généraux selon une description physique des processus biologiques. En partant de d'Arcy Thompson, adepte d'une conception purement mathématique et physicaliste de la forme, le cours présente d'abord un modèle d'organisation tissulaire reposant sur une description thermodynamique de la surface cellulaire et sur le concept de tension de surface. Suit l'exposé d'un modèle de la dynamique de la morphogenèse identifiant les forces actives de pression hydrostatique (chez les plantes) et de contractilité (chez les animaux), et la résistance variable que lui opposent les forces d'adhérences intercellulaires et/ou l'élasticité de la paroi cellulaire.

\section{Cours 2 - Adhésion : du concept d'affinité aux modèles thermodynamiques}

Avec ce second cours débute l'approfondissement du thème central de l'adhérence intercellulaire qui soulève quatre questions majeures: l'origine évolutive de la multicellularité, l'émergence au cours du développement embryonnaire de la forme des tissus et organes, l'origine chimique des couplages moléculaires intercellulaires, et l'origine physique des forces de cohésion au sein d'un tissu. L'enquête historique du concept d'adhérence débute avec les notions d'affinité et de spécificité discutées respectivement par Johannes Holtfreter et Paul Weiss. Les expériences de dissociation, agrégation et de tri cellulaire chez les éponges puis les amphibiens sont l'occasion du passage de la notion qualitative d'affinité au concept quantitatif, thermodynamique d'adhérence. L'hypothèse d'adhérence différentielle proposée par Steinberg est discutée en détail, entre réserves de fond exposées par A. Harris et apparente validation expérimentale après la découverte des molécules d'adhérence.

\section{Cours 3 - Adhésion : couplages intra- et extracellulaires}

Le troisième cours développe les éléments permettant de comprendre comment l'adhérence, propriété macroscopique intercellulaire, émerge des interactions moléculaires à la surface cellulaire. Nous explorons la relation entre, d'un côté, la biochimie et la biophysique des forces de liaison à l'échelle moléculaire et, de l'autre, les forces d'adhérence cellulaires. Dans un premier temps, le cours caractérise les interactions moléculaires extracellulaires, les éléments structuraux et dynamiques des liaisons cis- et trans- entre cadhérines. Il ressort de ces analyses qu'il s'avère impossible de prédire adéquatement l'énergie d'adhésion de la seule affinité d'interaction intermoléculaire à la surface des cellules.

Dans un second temps, les interactions dynamiques avec l'actine corticale sont abordées en évoquant successivement en quoi celles-ci affectent la dynamique des cadhérines à la surface, les forces d'adhérence intercellulaire et le comportement macroscopique de tri cellulaire. Ainsi, l'adhérence intercellulaire émerge de couplages dynamiques extracellulaires (entre cadhérines), et intracellulaires (avec l'actine corticale).

Pour finir, le cours adopte une perspective évolutive sur l'origine des mécanismes d'adhérence assurés par les cadhérines, en tant qu'ils impliquent des couplages intraet extracellulaires. Ce faisant, nous présentons une nouvelle hypothèse d'émergence de la multicellularité. 


\section{Cours 4 - Adhésion : un processus actif dissipatif, régulé}

Le quatrième cours clôt, momentanément, le chapitre sur l'adhérence. À la lumière du schéma moléculaire du cours précédent, nous développons le caractère hors équilibre de l'adhérence, son origine et ses implications fonctionnelles.

S'appuyant sur l'existence de couplages intermoléculaires dans le domaine extracellulaire et au sein du cortex intracellulaire, le cours présente tout d'abord un modèle de l'adhérence comme processus actif. En témoigne tout d'abord l'organisation des cadhérines en petits agrégats, révélant les contributions des couplages avec le cortex d'actine et de l'endocytose. Par ailleurs, la mécanosensation et la mécanotransduction par les Cadhérines témoignent de l'action des forces contractiles sur l'adhérence : stabilisation et renforcement des interactions au sein des complexes d'adhérence, adaptation à l'environnement mécanique d'une cellule, etc.

La dissipation énergétique au sein des complexes d'adhérence est traitée dans un second temps. Bien que ce sujet soit peu développé dans la littérature, le cours expose les raisons d'étudier cette notion centrale. Les processus dissipatifs assurent l'irréversibilité des interactions adhérentes et leur dynamique incessante aux échelles supramoléculaires et cellulaires. La dissipation repose sur l'existence conjointe d'un grand nombre d'interactions moléculaires de faible affinité, rapidement renouvelées et échangées, et de processus actifs qui orientent la dynamique des interactions. La caractérisation des modes de dissipation et des échelles de temps associées constituent un enjeu majeur des recherches sur le sujet.

Le cours se termine avec une vision synthétique de l'adhérence soulignant les principes communs opérant dans le cadre de l'adhérence intercellulaire, traitée dans ces cours (cadhérines) et de l'adhérence cellule-substrat (intégrines).

\section{Cours 5 - Tension cellulaire : tension de membrane}

Ce cinquième cours aborde le second thème central développé cette année, à savoir celui de la tension cellulaire. La tension cellulaire est en effet le moteur principal de la plasticité des tissus, qu'il s'agisse de tissus dans lesquels les cellules non cohésives migrent ou de tissus plus cohérents, comme les épithélia dans lesquels les interfaces cellulaires adhérentes sont constamment remodelées.

Nous partons du concept de tension de surface comme paramètre physique qui détermine certaines formes ou configurations cellulaires selon des modèles statiques de nature thermodynamique. Il aborde ensuite la forme cellulaire comme solution stationnaire ou stable d'un système dynamique. Dans ce cadre, il présente les contributions respectives de la tension membranaire et de la tension corticale. Le cours se donne pour objet de comprendre l'origine de la tension membranaire et de présenter aussi bien les méthodes quantitatives pour mesurer celle-ci que les modèles physiques permettant d'en définir les composantes. Sont soulignées en particulier la part de la tension dans le plan de la membrane stricto sensu, et celle de la tension due à l'adhésion entre l'actine corticale et la membrane plasmique.

Dans un second temps, le cours explore les implications d'un modèle de membrane fluide et inélastique dont la tension est généralement uniforme à la surface de la cellule. La tension de membrane dépend, d'un côté, des interactions dynamiques avec l'actine, de l'autre, de réservoirs dynamiques de membrane, notamment sous la forme d'invaginations. Nous montrons en quoi les interactions réciproques entre la membrane et l'actine assurent l'homéostasie de la tension de membrane en dépit des 
perturbations que peut connaître une cellule. Par ailleurs, l'étude de la migration cellulaire illustre de manière exemplaire en quoi la tension de membrane peut être un paramètre de contrôle de la physiologie cellulaire couplant et coordonnant des parties éloignées de la cellule et déterminant aussi bien sa forme que sa mobilité.

\section{Cours 6 - Tension cellulaire : tension corticale}

Le dernier cours de cette année porte sur le second volet de la tension cellulaire, à savoir la tension corticale. Elle émerge de l'activité contractile des moteurs moléculaires de type Myosine, s'associant aux filaments d'actine du cortex cellulaire.

Nous développons tout d'abord les propriétés remarquables des moteurs Myosine, des mécanoenzymes complexes qui suivent un cycle cinétique entraîné par l'ATP grâce à l'utilisation de l'énergie libre de liaison et d'hydrolyse de l'ATP, et de dissociation des produits d'hydrolyse, phosphate inorganique et ADP. La régulation cinétique des Myosines détermine le rapport cyclique du moteur, une mesure de la fraction du cycle associée à la génération de force mécanique. Les mécanismes d'ajustement de cette grandeur sont développés, où l'on voit notamment le rôle des régulations biochimiques de Myosine-II, et celui des forces mécaniques.

La seconde partie du cours aborde alors la question centrale de l'émergence des forces de tension dans un réseau entraîné par les forces contractiles de Myosine-II. Les contributions conjointes des cross-linkers d'actine, de l'activité de Myosine-II, de son organisation en minifilaments, de la longueur et de l'organisation spatiale des filaments d'actine sont évaluées à la lumière d'études récentes conjuguant mesure de paramètres physico-chimiques, perturbations et modélisation.

Nous considèrons alors les propriétés macroscopiques des réseaux d'actomyosine, notamment les propriétés viscoélastiques : d'un côté, leur comportement élastique sur de courtes échelles de temps et, de l'autre, leur comportement fluide sur de plus grandes échelles de temps donnant notamment naissance à des écoulements. Ces profiles dynamiques et les motifs spatiaux d'activation de la tension corticale sont à l'origine d'une grande diversité de formes et comportements dynamiques à l'échelle cellulaire, comme on le voit notamment au cours de la division et la migration de cellules.

Pour finir, la contribution de la tension corticale aux tensions interfaciales dans des cellules adhérentes est évaluée, en revenant au concept d'adhérence différentielle vu plus tôt cette année. Il ressort de ces études que la tension corticale a une contribution quantitative prépondérante à l'organisation et à la dynamique des interfaces cellulaires, par rapport aux forces d'adhérence stricto sensu.

Ce constat, ainsi que la nécessité de penser l'adhésion comme un système actif, dissipatif (cours 4), appellent une meilleure compréhension de l'interdépendance entre adhésion et tension cellulaire, de leur intégration aux contacts cellulaires et des processus de contrôle et d'auto-organisation de nature mécanochimique dont ils sont l'objet. Ce thème formera la trame du cours de l'an prochain, poursuivant le thème de la mécanique de la morphogenèse, en intégrant les échelles cellulaires et tissulaires au cours du développement.

\section{Colloque - MéCANIQUe CELlulaire et tissulaiRe (10 avril 2018)}

- Gijsje Koenderink (Amolf, Amsterdam): «Cell and extracellular matrix mechanics across scales: From molecules to supramolecular networks »; 
- Alexander Bershadsky (MBI Singapore) : «Self-organization of actin networks and cell morphogenesis »;

- Carl-Philip Heisenberg (IST, Vienne) : «Ooplasmic segregation in zebrafish »;

- Alpha Yap (Brisbane) : «Listening to forces: How mechanosensing makes cells into tissue ";

- Xavier Trepat (IBEC, Barcelone) : «Mechanics of cell migration, division and folding in epithelial sheets »;

- Sally Horne-Badovinac (université de Chicago) : «Going in circles gets you somewhere: Signaling mechanisms controlling collective cell migration »;

- Yohanns Bellaïche (Institut Curie, Paris) : «Mitosis and morphogenesis »;

- Kinneret Keren (Technion - Israel Institute of Technology) : «Mechanical aspects of hydra morphogenesis " ;

- Pierre-François Lenne (IBDM, Marseille) : «Shaping cell contacts during tissue morphogenesis »;

- Bénédicte Sanson (Cambridge): «Actomyosin supracellular cables and orientation of cell division in vivo »;

- Frank Jülicher (MPI PKS, Dresde) : « Shaping a fly wing »;

- Amy Shyer (Berkeley): «Cellular self-organization initiates skin form and fate $»$.

\section{Publications}

BanerJee D.S., Munjal A., Lecuit T. et Rao M., «Actomyosin pulsation and flows in an active elastomer with turnover and network remodeling », Nature Communications, vol. 8, $\mathrm{n}^{\mathrm{o}}$ 1, 2017, p. 1121, DOI : 10.1038/s41467-017-01130-1.

Clément R., Dehapiot B., Collinet C., LeCuit T. et Lenne P.-F., « Viscoelastic dissipation stabilizes cell shape changes during tissue morphogenesis ", Current Biology, vol. 27, $\mathrm{n}^{\circ}$ 20, 2017, p. 3132-3142.e4, DOI : 10.1016/j.cub.2017.09.005.

Jha A., van ZANTEN T.S., PhilipPe J.-M., MAYOR S. et LeCUit T., « Quantitative control of GPCR organization and signaling by endocytosis in epithelial morphogenesis ", Current Biology, vol. 28, nº 10, 2018, p. 1570-1584.e6, DOI : 10.1016/j.cub.2018.03.068.

LECUIT T., Dynamiques du vivant, Paris, Collège de France/Fayard, coll. « Leçons inaugurales du Collège de France », vol. 272, 2018 ; édition numérique : Collège de France, 2018, DOI : 10.4000/books.cdf.5781, https://books.openedition.org/cdf/5787.

Lecuit T. et Mahadevan L., "Morphogenesis one century after On Growth and Form », Development, vol. 144, n 23, 2017, p. 4197-4198, DOI : 10.1242/dev.161125. 
